\title{
PM2.5 concentration prediction in Lanzhou, China, using hyperchaotic cuckoo search--extreme learning machine
}

\author{
Wenjing Yang \\ Lanzhou University \\ Juan Du ( $\sim$ duj@lzu.edu.cn ) \\ Lanzhou University \\ Bowen Jia \\ Sichuan University \\ Ling Yang \\ Lanzhou University \\ Renze Dong \\ National University of Defense Technology \\ Xinyi Li \\ Southwest Jiaotong University
}

\section{Research Article}

Keywords: PM2:5 prediction, Hyperchaotic system, Cuckoo search, Extreme learning machine

Posted Date: February 11th, 2022

DOI: https://doi.org/10.21203/rs.3.rs-1314928/v1

License: (c) (i) This work is licensed under a Creative Commons Attribution 4.0 International License.

Read Full License 


\title{
$\mathrm{PM}_{2.5}$ concentration prediction in Lanzhou, China, using hyperchaotic cuckoo search - extreme learning machine
}

\author{
Wenjing Yang ${ }^{1}$, Juan $\mathrm{Du}^{1 *}$, Bowen $\mathrm{Jia}^{2}$, Ling Yang ${ }^{1}$, Renze \\ Dong ${ }^{3}$ and Xinyi li $^{4}$
}

${ }^{1 *}$ School of Information Science and Engineering, Lanzhou University, Lanzhou, 730000, China.

${ }^{2}$ Business School, Sichuan University, Chengdu, 610065, China. ${ }^{3}$ National University of Defense Technology, Changsha, 410000, China.

${ }^{4}$ Southwest Jiaotong University, chengdu, 614202, China.

*Corresponding author(s). E-mail(s): duj@lzu.edu.cn;

Contributing authors: yangwj2020@lzu.edu.cn;

j931427017@outlook.com; lingyang@lzu.edu.cn;

2394488622@qq.com; priscilla.lixinyi@foxmail.comrsity;

\begin{abstract}
Recently, Northwest China has been suffered $\mathrm{PM}_{2.5}$ pollution. Through a case study of Lanzhou city, capital of Northwest China's Gansu province, a hyperchaotic cuckoo search-extreme learning machine (HCCS-ELM) approach is proposed to establish a $\mathrm{PM}_{2.5}$ concentration prediction model. The cuckoo search is used to generate the number of hidden layer neurons in ELM. The hyperchaotic system is innovatively introduced to improve the accuracy of the algorithm with its good randomness and convergence. HCCS-ELM has a similar memory footprint as ELM, while experiments using observed data from four monitoring stations in Lanzhou during 2016 to 2018 show that HCCS-ELM has higher accuracy in $\mathrm{PM}_{\mathbf{2 . 5}}$ hourly and daily concentration prediction.
\end{abstract}

Keywords: $\mathrm{PM}_{2.5}$ prediction, Hyperchaotic system, Cuckoo search, Extreme learning machine 


\section{Introduction}

$\mathrm{PM}_{2.5}$ is still one of the environmental problems in China, and Lanzhou, as the most important industrial city in China, is facing more serious $\mathrm{PM}_{2.5}$ pollution. And artificial neural network has been widely used in air pollution prediction. Researchers have developed several $\mathrm{PM}_{2.5}$ concentration prediction models such as statistical and machine learning methods $[1,2]$. Recently, the performance of compound models has considerably increased with the development of computer hardware and software technologies, which has attracted significant attention in developing various prediction models. For example, convolutional back-propagation neural networks [3, 4], image contrast-sensitive features and weighted bagging based neural network can be integrated to predict air pollution [5]. However, a hybrid convolutional neural network combined with the high-level features extracted from the convolutional layer with ground-truth $\mathrm{PM}_{2.5}$ can learn haze-relevant classified mapping and train support vector regression, indicating cheapness, quickness, and convenience [6]. Besides, neural networks can derive a kind of multivariate Bayesian uncertainty processor to predict the probabilities of $\mathrm{PM}_{2.5}$ [7] or develop an air quality early-warning system by phase-space reconstruction and multi-objective optimization [8]. In addition, the AOD-PM $\mathrm{P}_{2.5}$ model uses a combination of multiple linear regression, back propagation neural networks, classification and regression trees, and random forest estimation methods to predict $\mathrm{PM}_{2.5}$ concentrations, and improves accuracy in East China by adding meteorological elements that vary with time and height [9].

Deterioration of air quality has affected people in China [10-12]. Chinese researchers [13] proposed a hybrid model that can rapidly estimate particulate pollution based on a data-driven artificial neural network (ANN). At the regional level, Zhao et al. [14] used recurrent neural networks to analyze hourly air quality in the Northwest of China. Besides, Wang et al. [15] exploited an $\mathrm{ANN}$ to predict and analyze the $\mathrm{PM}_{2.5}$ in Chongqing, China. In terms of small-scale predictions, Tong et al. [16] and Xu et al. [17] investigated machine learning to analyze $\mathrm{PM}_{2.5}$ in the households in Hong Kong and near the Beijing railway station, respectively.

Air quality is an environmental and major survival issue [18, 19]. As an important city in Northwest China, Lanzhou has been plagued because of air pollution for a long time. The $\mathrm{PM}_{2.5}$ concentration in Lanzhou far exceeds the Chinese warning line. In March 2021, Lanzhou encountered a severe sandstorm. In Figure 1, the red dotted line represents the $\mathrm{PM}_{2.5}$ concentration limit of China. While the blue one represents the $\mathrm{PM}_{2.5}$ concentration in Lanzhou, which severely exceeds China's standard in recent years. The $\mathrm{PM}_{2.5}$ concentration is dynamic, related to time series, and presents seasonal changes. Thus, an accurate prediction of $\mathrm{PM}_{2.5}$ concentration is of great significance for strengthening the prevention and control of air pollution [20].

Most of the existing algorithms are aimed at the prediction of short-term or long-term pollutant concentration. There is currently no algorithm that can 


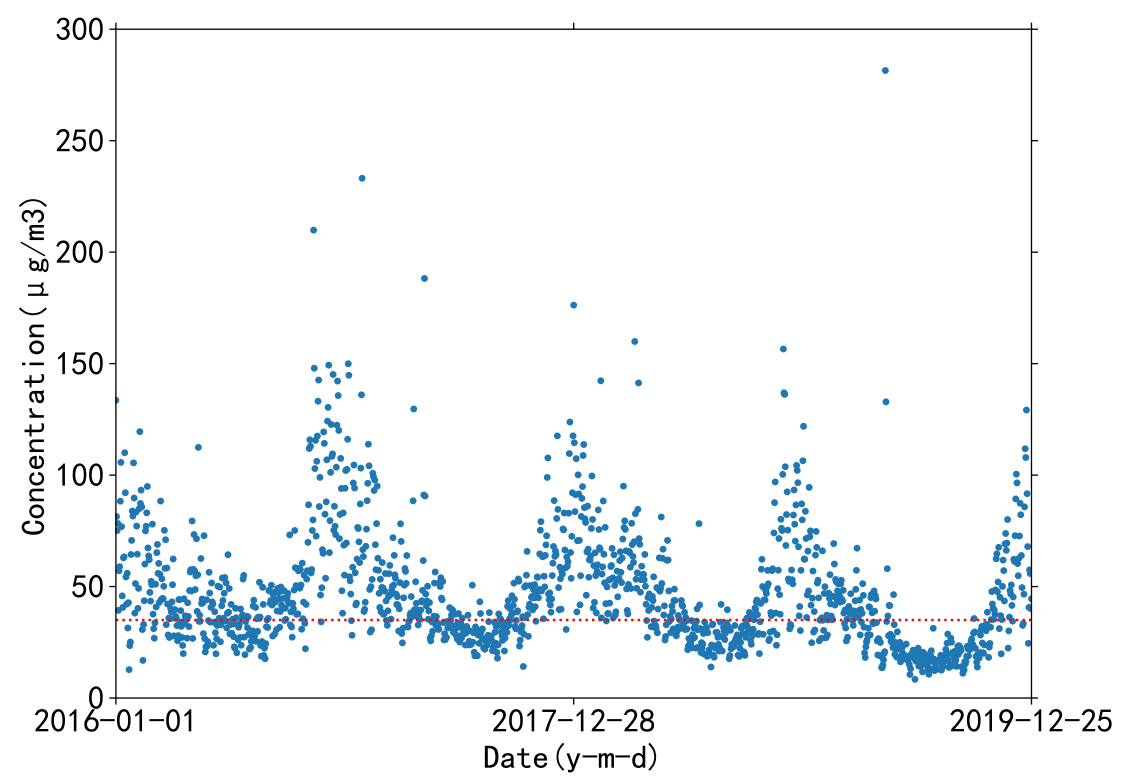

Fig. 1: 2016-2019 $\mathrm{PM}_{2.5}$ concentration in Lanzhou, China

perform well in long-term and short-term $\mathrm{PM}_{2.5}$ forecasts. The inherent neural network is complicated to calculate, occupies a lot of system resources, and has poor prediction accuracy for $\mathrm{PM}_{2.5}$ concentration. There is a correlation between $\mathrm{PM}_{2.5}$ concentration and time series, so we use an extreme learning machine (ELM) method to predict $\mathrm{PM}_{2.5}$ concentration. Avoiding manual adjustment, cuckoo search (CS) algorithm can be provided to calculate the optimal number of hidden layer neurons of ELM. For the good convergence and randomness of hyperchaotic systems [21], its join can reduce the computational cost of CS algorithm and improve the accuracy of ELM [22]. Inspired by the above, this paper proposes hyperchaotic CS-ELM (HCCS-ELM) to predict the $\mathrm{PM}_{2.5}$ concentration in Lanzhou. The proposed HCCS-ELM is validated using the measured data selected from four monitoring stations in Lanzhou. The results show that HCCS-ELM has good performance in predicting $\mathrm{PM}_{2.5}$ hourly or daily concentration in Lanzhou. Compared with ELM, HCCS-ELM utilizes a similar memory footprint, and it is conducive to the accurate and efficient prediction of $\mathrm{PM}_{2.5}$ concentration. Besides, it can reduce the cost and the time required for prediction under the condition of ensuring the same accuracy. By accurately predicting the $\mathrm{PM}_{2.5}$ concentration, preventive and effective measures can be taken in advance to minimize the losses caused by heavy pollution weather [23]. 


\section{Method}

\subsection{Study Area}

Lanzhou is the capital city of Gansu, located at $36^{\circ} 03^{\prime} \mathrm{N}$ and $103^{\circ} 40^{\prime} \mathrm{E}$. The terrain is high in the southwest and low in the northeast. Surrounded by mountains in the north and south of the urban area, the Yellow River passes through the city, forming a narrow long valley between gorges and basins. The frequency of calm winds and temperature inversion in the Lanzhou River Basin is high, which is not conducive for the diffusion of endogenous pollutants. And the transfer of external dust in the surrounding area results in severe $\mathrm{PM}_{2.5}$ pollution.

\subsection{Air Quality Monitoring and Meteorological Data}

The meteorological data comes from website (http://data.cma.cn/), and the pollution data comes from website (http://www.mee.gov.cn/). In this paper, four stations provide the data, which geographic positions are shown in Figure 2. Station 1 is located in Xigu District, Lanzhou City, next to Lanzhou Petrochemical, and has relatively low traffic. Station 2 is located around the city of Lanzhou, with low traffic and no factories nearby. Stations 3 and 4 are located in the residential area of Lanzhou City, with heavy traffic and no factories nearby. The four stations do not affect the surrounding environment, which means they are hidden. For example, if people know where the stations are, they may go away, reducing traffic flow and resulting in inaccurate data. We select daily and hourly meteorological data such as wind direction, wind speed, temperature, relative humidity and pollutant $\left(\mathrm{CO}, \mathrm{SO}_{2}, \mathrm{NO}_{2}, \mathrm{O}_{3}\right.$ and $\mathrm{PM}_{2.5}$ ) data from 2016 to 2018 as research samples [24-26].

\subsection{Hyperchaotic System}

In this section, we adopt a hyperchaotic real system and its corresponding complex system to generate the input weights and hidden layer biases of HCCSELM to obtain better prediction results than models for comparison. The hyperchaotic real system is given as follows [22, 27]:

$$
\left\{\begin{array}{l}
\dot{\xi_{1}}=-a \xi_{1}+\xi_{2} \xi_{3}+\xi_{4}, \\
\dot{\xi_{2}}=-\xi_{1}+c \xi_{2}, \\
\dot{\xi_{3}}=\xi_{1} \xi_{2}-b \xi_{3}, \\
\dot{\xi_{4}}=d \xi_{1}+e \xi_{2},
\end{array}\right.
$$

where $a, b, c$ and $d$ are real constants, and $\xi_{1}, \xi_{2}, \xi_{3}$ and $\xi_{4}$ are real variables. The dots denotes derivatives with respect to time $t$. The corresponding hyperchaotic complex system is given by 


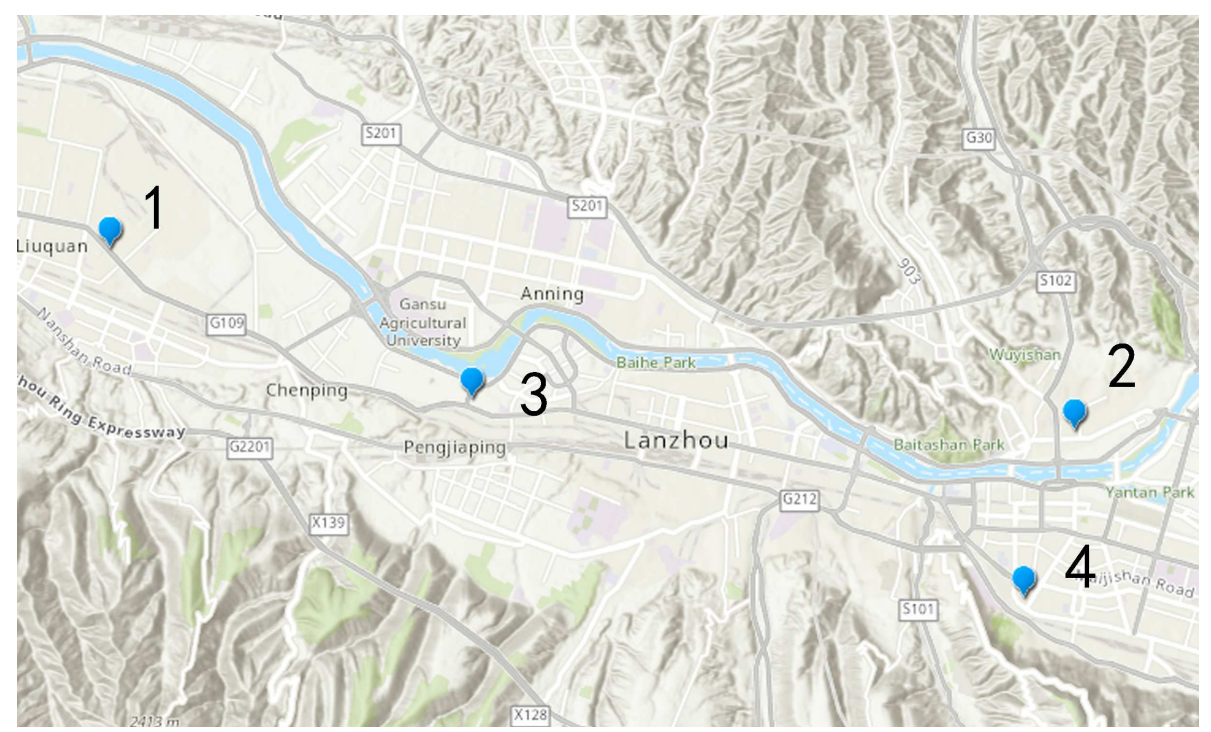

Fig. 2: Geographic positions of four stations in Lanzhou, China (From left to right are Station 1, 3, 4 and 2)

$$
\left\{\begin{array}{l}
\dot{\eta_{1}}=-a \eta_{1}+\eta_{2} \eta_{3}+\eta_{4} \\
\dot{\eta_{2}}=-\eta_{1}+c \eta_{2} \\
\dot{\eta_{3}}=\frac{1}{2}\left(\bar{\eta}_{1} \eta_{2}+\eta_{1} \bar{\eta}_{2}\right)-b \eta_{3} \\
\dot{\eta_{4}}=d \eta_{1}+e \eta_{2}
\end{array}\right.
$$

where $\eta_{1}=u_{1}+j u_{2}, \eta_{2}=u_{3}+j u_{4}, \eta_{3}=u_{5}$, and $\eta_{4}=u_{6}+j u_{7}$ are complex variables, and $j=\sqrt{-1}$. The over bar represents the complex conjugate of the variables. The real version of System (2) is given as follows:

$$
\left\{\begin{array}{l}
\dot{u_{1}}=-a u_{1}+u_{3} u_{5}+u_{6} \\
\dot{u_{2}}=-a u_{2}+u_{4} u_{5}+u_{7} \\
\dot{u_{3}}=-u_{1}+c u_{3} \\
\dot{u_{4}}=-u_{2}+c u_{4} \\
\dot{u_{5}}=u_{1} u_{3}+u_{2} u_{4}-b u_{5} \\
\dot{u_{6}}=d u_{1}+e u_{3} \\
\dot{u_{7}}=d u_{2}+e u_{4}
\end{array}\right.
$$

The hyperchaotic sequences are shown in Figure 3 for the $\xi_{3}-\xi_{2}-\xi_{1}$ and $\xi_{2}-\xi_{1}-\xi_{4}$ planes. 
the $\xi_{3}-\xi_{2}-\xi_{1}$ plane

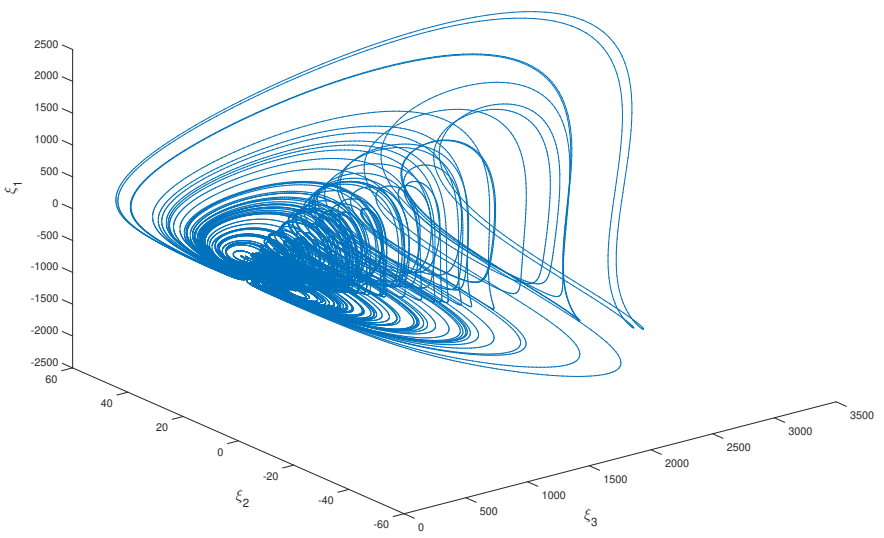

(a)

the $\xi_{2}-\xi_{1}-\xi_{4}$ plane

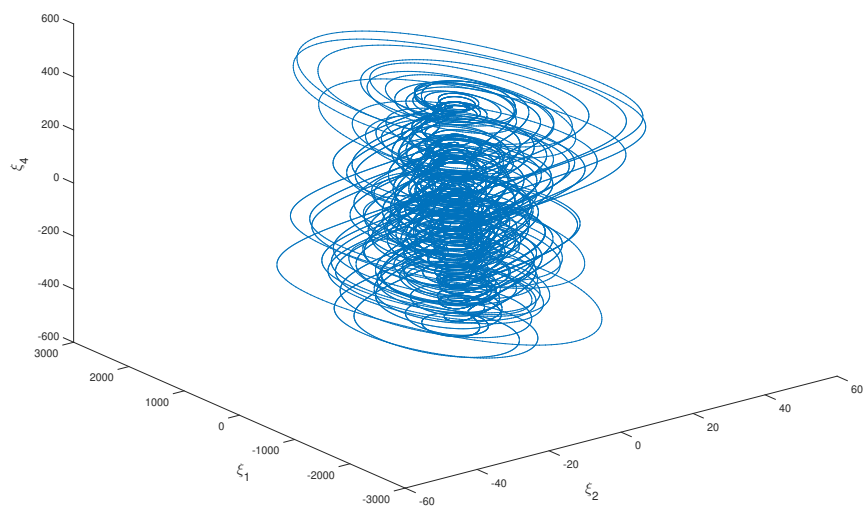

(b)

Fig. 3: Phase diagrams for System (1) in the (a) $\xi_{3}-\xi_{2}-\xi_{1}$ plane, (b) $\xi_{2}-\xi_{1}-\xi_{4}$ plane 


\subsection{Hyperchaotic Cuckoo Search}

The CS algorithm is a behavior simulation proposed by Yang et al. [28] based on the interesting breeding behavior such as brood parasitism of certain species of cuckoos. Meanwhile, they introduced the breeding behavior of cuckoos and characteristics of Lévy flights of some birds and fruit flies' trajectories. In nature, cuckoo birds fly in random or similar random ways to find the location of the nest suitable for laying eggs. The CS algorithm has three ideal conditions as the premise [28, 29]:

(1) Each cuckoo lays only one egg at a time, and randomly selects a nest location for hatching.

(2) In a randomly selected set of nests, the high-quality ones will be retained for the next generation.

(3) The number of available nests is fixed, and the probability of bird's nest owner finding the foreign egg is $P_{a} \in[0,1]$. When the owner finds a foreign cuckoo egg, he will throw it away or build a new nest.

Based on the above, the operation steps of cuckoo algorithm are as follows: (1) Setting parameters such as population size, searching space dimension, and maximum number of iterations. The position of the nest is initialized randomly as $X_{i}, i \in[1, n]$, and the objective function is defined as $f(x), x=\left[x_{1}, x_{2}, x_{n}\right]^{T}$, where $T$ denotes transpose.

(2) Calculating the objective function value of each nest location and comparing it to obtain the current optimal function value.

(3) Using hyperchaotic sequence to update the position and state of other nest except the optimal one, calculating the objective function value and comparing the obtained function value with the current optimal one. If it is better, the current optimal value is updated.

(4) After position updating, random numbers $r$ and $P_{a}$ are used for comparison. If $r>P_{a}$ is used, the nest position is randomly updated once. Otherwise, it remains unchanged.

(5) If the maximum number of iterations or search accuracy requirements are met, the next step will proceed, or it will go back to Step (3).

(6) Outputting the location of the globally optimal nest.

\subsection{Hyperchaotic Extreme Learning Machine}

The hyperchaotic ELM is divided into three parts: input, hidden and output layers. In the network, neurons of input, hidden and output layers are completely connected. Assume that the model has $n$ input layer neurons and its corresponding input has $n$ data variables, $j$ neurons in the hidden layer, and $m$ in the output layer so that its corresponding output has $m$ data variables [30]. $W$ is the connection weight matrix of input and hidden layers. It is represented using the following matrix: 


$$
W=\left[\begin{array}{cccc}
w_{11} & w_{12} & \cdots & w_{1 n} \\
w_{21} & w_{22} & \cdots & w_{2 n} \\
\vdots & \vdots & \ddots & \vdots \\
w_{l 1} & w_{l 2} & \cdots & w_{l n}
\end{array}\right]_{l \times n}
$$

where $w_{j i}$ is the connection weight between the $i^{\text {th }}$ input and the $j^{\text {th }}$ hidden neurons.

$\beta$ is the connection weight between the hidden and output layers as shown in Eq. 5:

$$
\beta=\left[\begin{array}{cccc}
\beta_{11} & \beta_{12} & \cdots & \beta_{1 m} \\
\beta_{21} & \beta_{22} & \cdots & \beta_{2 m} \\
\vdots & \vdots & \ddots & \vdots \\
\beta_{l 1} & \beta_{l 2} & \cdots & \beta_{l m}
\end{array}\right]_{l \times m}
$$

where $\beta_{k j}$ is the weight from the hidden neuron $j$ to the output neuron $k$. Both $W$ and the status of neuron threshold in the hidden layer are generated using the above mentioned hyperchaotic system.

Hyperchaotic sequences generated using the above hyperchaotic functions are used to replace the hidden layer threshold matrix randomly generated using the ELM. The running steps of hyperchaotic ELM are as follows:

(1) Importing data and starting training.

(2) Generating the connection weight matrix between the input and hidden layers randomly.

(3) Generating threshold matrix of hidden layer using the hyperchaotic function.

(4) Calculating the connection weight matrix between the hidden and output layers using the least square method.

(5) Importing the test data into the hyperchaotic ELM and outputting the prediction results after the training.

\subsection{HCCS-ELM}

Figure 4 shows the structure diagram of HCCS-ELM. First, the training data are input. Then, the chaos equation runs to generate the hyperchaotic sequence. Sequence 1 is selected to replace the Lévy flight in the cuckoo algorithm. It realizes the jump by randomly selecting the value in sequence without repetition. Sequence 2 is selected to replace the randomly generated connection weight matrix of the input and hidden layers. Sequence 3 is used to generate the hidden layer threshold matrix in the ELM. The improved cuckoo algorithm is used to traverse the sample data to generate the number of hidden layer neurons in the ELM. After the training process of ELM, the test data is imported, and the prediction results are obtained.

To reduce the system computational cost, the hyperchaotic system is used to generate a sequence instead of the Lévy flight. The hyperchaotic system 


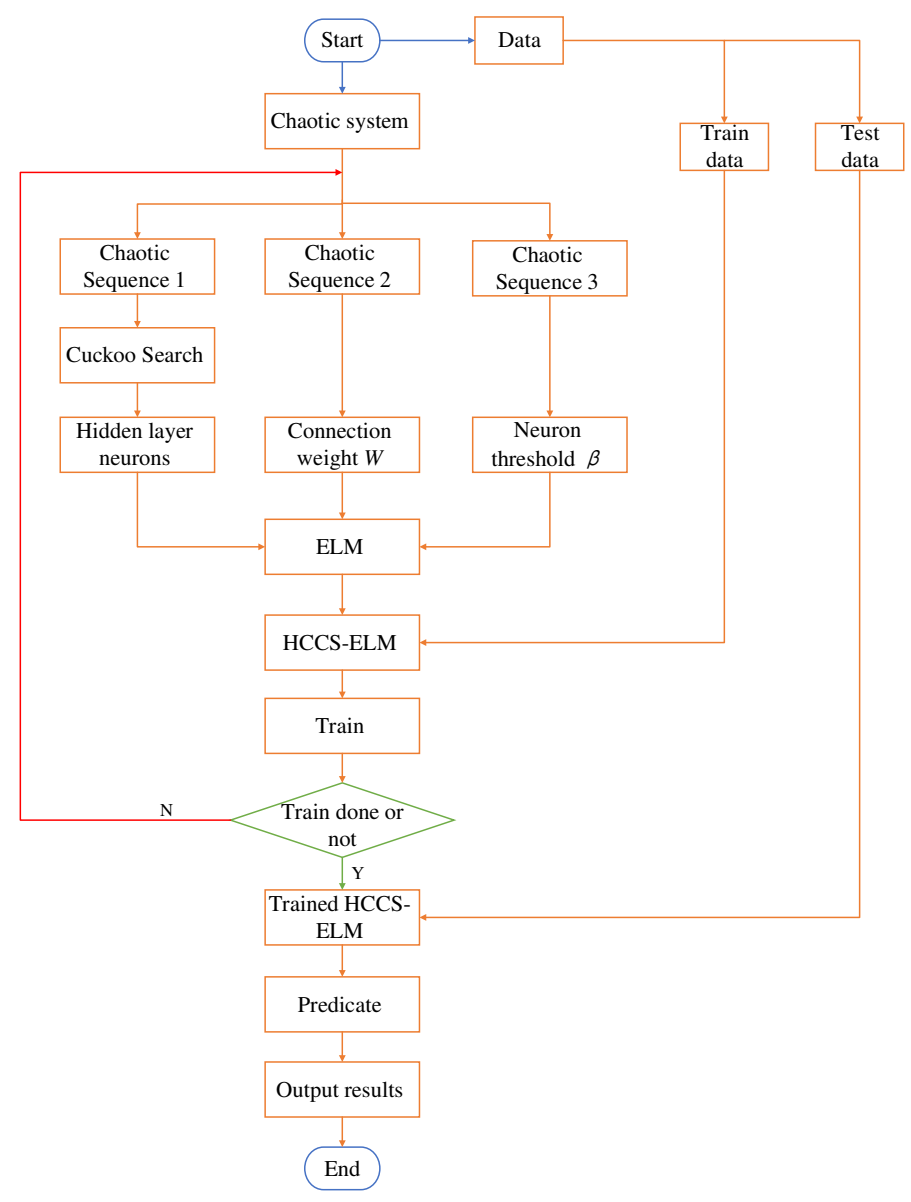

Fig. 4: System chart of HCCS-ELM

is only required to generate a sequence once, and then each iteration randomly selectes a value from the sequence, which greatly reduces the resource occupation. Besides, CS is exploited to traverse the samples to generate the number of hidden layer neurons, which could effectively improve the algorithm's adaptability and avoid repeated parameter adjustments. Considering the convergence phenomenon of chaos, the hyperchaotic sequence is used to replace the hidden layer threshold matrix in ELM, and regression could be understood as a convergence phenomenon. Thus, the convergent sequence is used to replace the random one, which improves the ELM's accuracy and generates highly accurate results in $\mathrm{PM}_{2.5}$ concentration prediction, saving the use of system resources. 
Table 1: The amount of data on daily pollutant concentrations of four stations in each season

\begin{tabular}{ccccc}
\hline Amount Season & Spring & Summer & Autumn & Winter \\
\hline Station & & & & \\
\hline Station 1 & 183 & 186 & 168 & 178 \\
Station 2 & 183 & 186 & 183 & 178 \\
Station 3 & 183 & 186 & 183 & 178 \\
Station 4 & 183 & 186 & 183 & 178 \\
\hline
\end{tabular}

\section{Experiments and Results}

\subsection{Long-term Prediction}

This study uses HCCS-ELM, back-propagation (BP), multiple linear regression (MLR), ELM, Long Short-Term Memory(LSTM) and Prophet to predict the $\mathrm{PM}_{2.5}$ concentration of four observing stations. Besides, it compares and analyzes the results similar to that of [31]. Seventy percent of the historical observation data are used to train the model, and the remaining $30 \%$ data are used to test the model. Since the $\mathrm{PM}_{2.5}$ concentration varies across seasons, so that it is predicted for different seasons. For the instrument failure and other irresistible making historical observations incompletable, the invalid data are deleted if one or more kinds of variables are missing. However, special days, such as rainy days, holidays, or night time are not a criterion for filtering data. Thus, only two months in each season are selected for prediction. Table 1 presents the amount of filtered data. There are 500 samples in the training set of $\mathrm{PM}_{2.5}$ prediction at station one, and 215 samples in the test set. There are 511 samples in the training set of $\mathrm{PM}_{2.5}$ predictions of station 2,3 and 4 , and 219 samples in the test set. Eight impact factors are located in the input layer. The predicted $\mathrm{PM}_{2.5}$ concentration is output in the output layer. Both HCCS-ELM and ELM have the same parameters, which are sigmoid function, "regression" model, and 10 hidden layer neurons. However, the BP network has 10 hidden layer neurons, 100 epochs, 0.1 learning rate, and 0.4 goals. All parameters of the six models remain unchanged during the experiment. The root mean squared error ( $R M S E)$ and the coefficient of determination $R^{2}$ are selected as the evaluation criteria. The formulas of $R M S E$ and $R^{2}$ are given as follows:

$$
\begin{gathered}
\text { RMSE }=\sqrt{\frac{\sum_{i=1}^{n}\left(X_{o b s, i}-X_{\text {model }, i}\right)^{2}}{n}} \\
R^{2}=1-\frac{R M S E}{V a r}
\end{gathered}
$$

where $n$ is the number of samples, $X_{o b s, i}$ is the observed value of $\mathrm{PM}_{2.5}$ concentration, $X_{\text {model }, i}$ is the predicted value of $\mathrm{PM}_{2.5}$ concentration, and $\operatorname{Var}(\cdot)$ is the variance. The smaller the $R M S E$ and the closer $R^{2}$ is to 1 , it indicates that the prediction effect is better. 
MLR, LSTM and Prophet are run with Python. Figure 5 shows the HCCS-ELM prediction results. Tables 2 and 3 present the $R M S E$ and $R^{2}$ of HCCS-ELM, ELM, BP, MLR, LSTM and Prophet. The analysis of the above chart show that the RMSE of HCCS-ELM are smaller than the other five models, and the $R^{2}$ are larger than the other models, which could predict the concentration and the trend of $\mathrm{PM}_{2.5}$ more accurately. Table 2 shows the results obtained by the daily concentration from 2016 to 2017 as the training set and the 2018 daily concentration as the test set; Table 3 uses the first two months of each quarter in 2018 as the training set, and the last month as the test set.

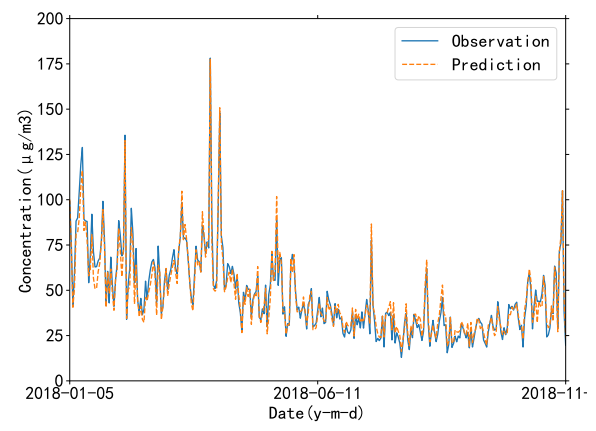

(a) Station 1

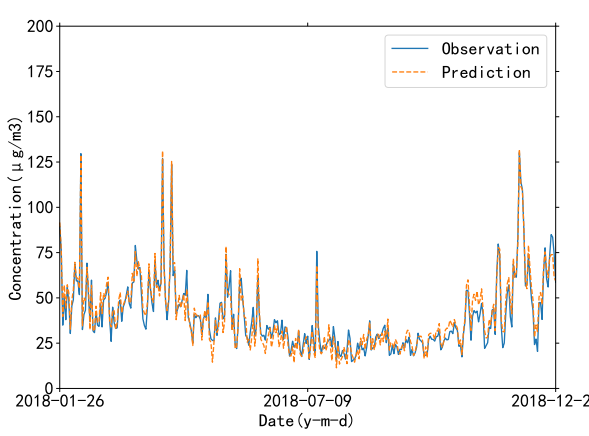

(c) Station 3

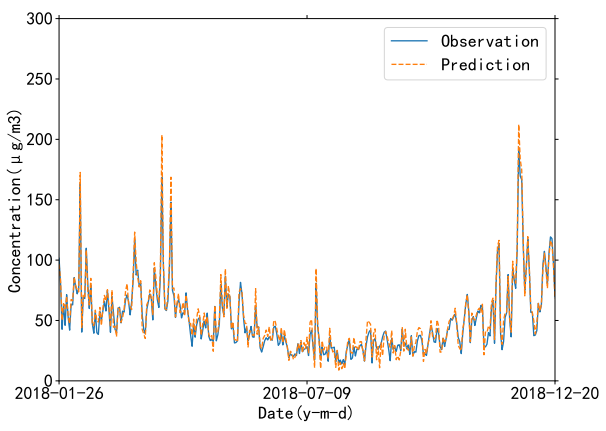

(b) Station 2

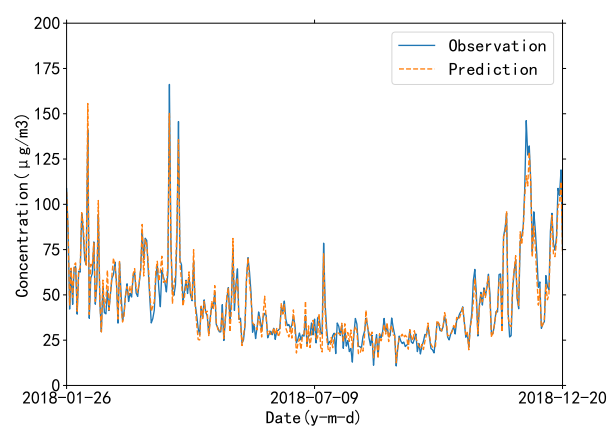

(d) Station 4

Fig. 5: Long-term prediction results of HCCS-ELM (Year) 
Table 2: $R M S E$ and $R^{2}$ values by station and method (Year)

\begin{tabular}{|c|c|c|c|c|c|c|c|c|c|c|c|c|}
\hline & \multicolumn{6}{|c|}{$R M S E$} & \multicolumn{6}{|c|}{$R^{2}$} \\
\hline & HCCS-ELM & ELM & $\mathrm{BP}$ & MLR & LSTM & Prophet & HCCS-ELM & ELM & $\mathrm{BP}$ & MLR & LSTM & Prophet \\
\hline Stati & 4.6255 & 9.3645 & 15.7328 & 17.7866 & 16.5247 & 16.5238 & 0.9627 & 0.8842 & 0.6079 & 0.4131 & 0.4858 & 0.4929 \\
\hline Station 2 & 5.7935 & 11.8354 & 17.8923 & 21.3147 & 91 & & & 97 & 0.6627 & 0.4296 & 0.5626 & 0.4366 \\
\hline Station 3 & 4.6431 & 8.9009 & 24.6076 & 15.7897 & 13.6778 & & & 0.8244 & 0.6607 & 0.3524 & 0.5006 & 0.3601 \\
\hline Station 4 & 4.7172 & 8.4336 & 20.3576 & 19.1319 & 16.2429 & 17.6423 & 0.9625 & 0.8931 & 0.6853 & 0.3979 & 0.5423 & 0.4588 \\
\hline
\end{tabular}


Table 3: $R M S E$ and $R^{2}$ values by station and method (Season)

\begin{tabular}{|c|c|c|c|c|c|c|c|c|c|c|c|c|c|}
\hline & \multicolumn{6}{|c|}{$R M S E$} & \multicolumn{6}{|c|}{$R^{2}$} \\
\hline & & HCCS-ELM & ELM & BP & MLR & LSTM & rophet & HCCS-ELM & ELM & BP & MLR & LSTM & Prophet \\
\hline \multirow{4}{*}{ Spring } & Station 1 & 5.0233 & 9.1717 & 19.1538 & 20.9775 & 16.7506 & 18.1760 & 0.9371 & 0.8384 & 0.6513 & 0.1443 & 0.2827 & 0.1386 \\
\hline & Station 2 & 7.1416 & 9.7955 & 13.7006 & 26.1891 & 17.3320 & 19. 0321 & 0.8987 & 0.8513 & 0.7429 & 0.0236 & 0.2708 & 0.1075 \\
\hline & Station 3 & 4.8586 & 15.2012 & 10.1559 & 18.5039 & 14.5123 & 15.4039 & 0.9236 & 0.6557 & 0.8090 & 0.0760 & 0.2200 & 0.1156 \\
\hline & Station 4 & 5.2844 & 14.7320 & 8.8194 & 22.7076 & 16.6815 & 17.3568 & 0.9 & 0.7330 & 0.8115 & 0.0135 & 0.2602 & 0.1880 \\
\hline \multirow{4}{*}{$\mathrm{mm}$} & ion 1 & 3.4992 & 9359 & 5.1225 & 10.7228 & 10.6803 & 9.9579 & & .7087 & 0.8219 & 0.0551 & .0420 & 1275 \\
\hline & 100 & 6244 & 8195 & 7.2272 & 14.7589 & 11.8178 & & & 0.7533 & 0.7443 & 0.0161 & 0.0637 & .1125 \\
\hline & Station 3 & .2680 & 5.7048 & 4.3199 & 10.1234 & 8.9888 & 8.1486 & 0.9360 & 0.7222 & 0.7889 & 0.0081 & 0.0007 & 0.1425 \\
\hline & Station 4 & 2.2604 & 4.5818 & 4.4368 & 11.1462 & 9.5097 & 8.7015 & 81 & 0.7903 & 0.8476 & 0.032 & 0.0127 & 0.1496 \\
\hline \multirow{4}{*}{ Autur } & Station 1 & 6.5007 & 11.3300 & 13.9920 & 13.7498 & 17.1170 & 17.4268 & 0.9106 & 0.7194 & 0.6930 & 0.5252 & 0.1574 & 0.1389 \\
\hline & Station 2 & 8.1300 & 13.2583 & 19.2205 & 29.0382 & 23.7319 & 24.6825 & 0.9543 & 0.8987 & 0.8456 & 0.4161 & 0.5688 & 0.5220 \\
\hline & tation 3 & 7.9112 & 13.8340 & 14.1030 & 21.6965 & 14.2278 & 15.3461 & 0.9164 & 0.7875 & 0.8581 & 0.3124 & 0.6511 & 0.5855 \\
\hline & Station 4 & 6.1445 & 14.5434 & 15.9177 & 21.5627 & 17.2101 & 18.1260 & 0.9555 & 0.7317 & 0.7966 & 0.4725 & 0.6487 & 0.6050 \\
\hline \multirow{4}{*}{ Winter } & Station 1 & 7.2779 & 12.7341 & 10.5278 & 17.0671 & 20.9554 & 20.6743 & 0.9457 & 0.7307 & 0.8011 & 0.5285 & 0.1664 & 0.1717 \\
\hline & Station 2 & 8.4257 & 20.8565 & 23.9630 & 19.5162 & 24.5324 & 22.5306 & 0.9271 & 0.7488 & 0.8243 & 0.5172 & 0.0012 & 0.1391 \\
\hline & Station 3 & 3.8454 & 12.7414 & 16.3453 & 17.1244 & 18.5874 & 17.4310 & 0.9590 & 0.7347 & 0.7582 & 0.2818 & 0.0216 & 0.1277 \\
\hline & Station 4 & 5.9452 & 13.5708 & 13.3386 & 22.1096 & 22.2022 & 20.4694 & 0.9344 & 0.7172 & 0.8402 & 0.3632 & 0.0277 & 0.1453 \\
\hline
\end{tabular}




\subsection{Short-term Prediction}

The short-term extremely high $\mathrm{PM}_{2.5}$ concentration is usually the object of the attention of the meteorological department. High $\mathrm{PM}_{2.5}$ concentration is harmful to human health. In this paper, data with $\mathrm{PM}_{2.5}$ concentrations exceeding China's national standard are selected, and the data division is consistent with the above. Figure 6 shows that HCSS-ELM is still efficient for prediction of short-term extremely high $\mathrm{PM}_{2.5}$ concentration. Due to the missing data, hourly concentration data is incomplete. In Figure 6, the test set is the hourly concentration of the date in the figure, and the training set is the effective value of the hourly concentration fourteen days before the test date.

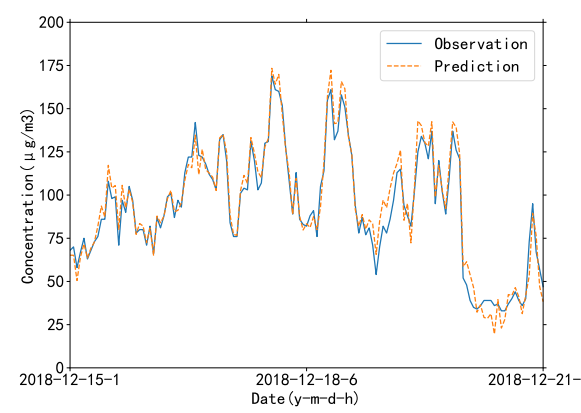

(a) Station 1

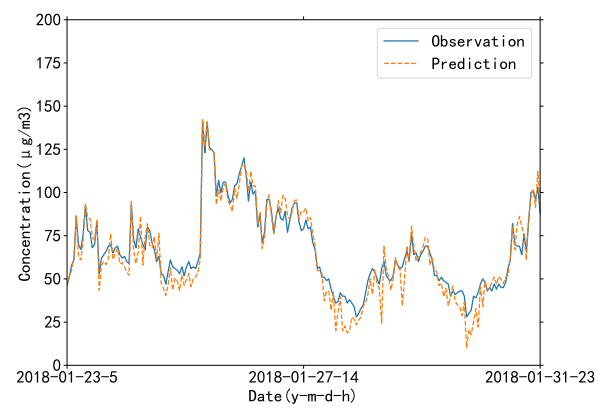

(c) Station 3

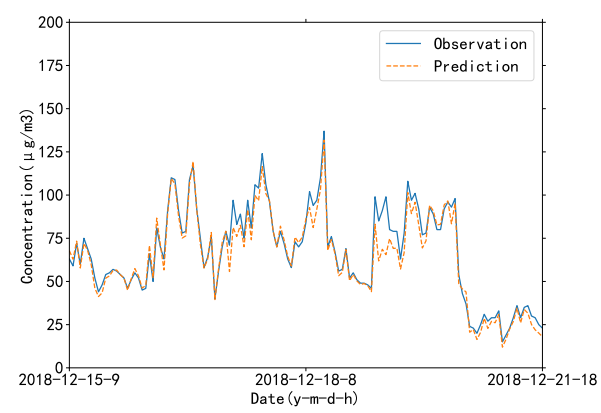

(b) Station 2

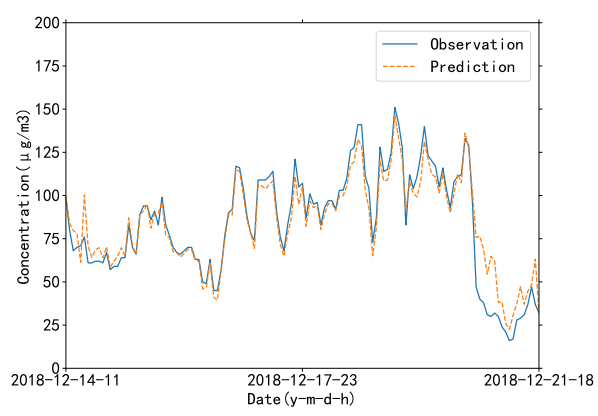

(d) Station 4

Fig. 6: Short-term prediction results of $\mathrm{PM}_{2.5}$ based on HCCS-ELM

\subsection{Performance Evaluation}

The running time and memory usage are used to evaluate the performance of HCCS-ELM and ELM. Tables 4 and 5 present the time and memory results. 
Table 4 shows the performance of the two algorithms in processing the daily concentration data of each station from 2016 to 2018, and Table 5 shows the performance of the two algorithms in processing the daily concentration data of four stations in each quarter. It shows that HCCS-ELM needs more running time than ELM, while they have similar occupied memory. That's because HCCS-ELM need to generate hyperchaotic sequences during operation. The differences in running time and memory usage of the same model can be attributed to the software.

Table 4: Performance comparison between HCCS-ELM and ELM (Year)

\begin{tabular}{lcccc}
\hline & \multicolumn{2}{c}{ Running time (s) } & \multicolumn{2}{c}{ Memory (MB) } \\
& HCCS-ELM & ELM & HCCS-ELM & ELM \\
\hline Station 1 & 2.2436 & 0.2049 & 2026 & 2004 \\
Station 2 & 2.2767 & 0.1598 & 2026 & 2004 \\
Station 3 & 2.2693 & 0.1683 & 2028 & 2003 \\
Station 4 & 2.2826 & 0.1716 & 2028 & 2013 \\
\hline
\end{tabular}

Table 5: Performance comparison between HCCS-ELM and ELM (Season)

\begin{tabular}{llcccc}
\hline \multirow{5}{*}{ Spring } & & \multicolumn{2}{c}{ Running time (s) } & \multicolumn{2}{c}{ Memory (MB) } \\
& & HCCS-ELM & ELM & HCCS-ELM & ELM \\
\hline \multirow{5}{*}{ Summer } & Station 1 & 2.1104 & 0.0222 & 2067 & 2113 \\
& Station 2 & 2.1132 & 0.0164 & 2074 & 2104 \\
& Station 3 & 2.3861 & 0.0165 & 2073 & 2083 \\
& Station 4 & 2.1071 & 0.0167 & 2072 & 2082 \\
\hline \multirow{5}{*}{ Autumn } & Station 1 & 2.1414 & 0.0188 & 2063 & 2067 \\
& Station 2 & 2.2904 & 0.0184 & 2062 & 2067 \\
& Station 3 & 2.1173 & 0.0204 & 2063 & 2066 \\
& Station 4 & 2.1473 & 0.0161 & 2061 & 2065 \\
\hline \multirow{5}{*}{ Winter } & Station 1 & 2.1230 & 0.0163 & 2081 & 2160 \\
& Station 2 & 2.1389 & 0.0162 & 2079 & 2161 \\
& Station 3 & 2.1251 & 0.0183 & 2078 & 2147 \\
& Station 4 & 2.1200 & 0.0228 & 2078 & 2147 \\
\hline & Station 1 & 2.1002 & 0.0227 & 2062 & 2072 \\
& Station 2 & 2.1172 & 0.0160 & 2060 & 2062 \\
& Station 3 & 2.4589 & 0.0171 & 2069 & 2069 \\
& Station 4 & 2.1337 & 0.0158 & 2076 & 2060 \\
\hline
\end{tabular}

\section{Discussion}

In March 2021, most parts of China suffered a severe sandstorm. From the perspective of environmental protection, people should improve the environmental protection system, adopt an environmentally friendly way of life, and insist on the harmonious coexistence between man and nature. For the improvement of the global ecological environment, it is necessary to set up and 
practice the concept that clear waters and green mountains are as valuable as mountains of gold and silver.

Machine learning has fast and accurate performance in prediction and is commonly used to predict the trend of pollutant concentration transformation. Accurate prediction of pollutant concentrations can help local governments take more effective preventive measures. Therefore, based on existing methods, this paper proposes the HCCS-ELM algorithm, which has the following advantages in analyzing $\mathrm{PM}_{2.5}$ concentrations in Lanzhou. First, HCCS-ELM simplifies the analysis process, while traditional numerical weather forecast methods are very complex and require a lot of calculations. And then, air quality forecasting cost is reduced. The proposed HCCS-ELM is similar to other neural networks in that it is independent of the complex relationship between the parameters and outputs. However, it depends on constant change of weights so that the parameters and outputs are closely related and tedious mathematical modeling is avoided. Second, HCCS-ELM has good generalization. Since there is no mathematical modeling process, combined with similar and available data, HCCS-ELM can theoretically be applied to all cities in China that set up air quality monitoring stations without being affected by differences in geographical environment and the level of economic development. Third, considering the HCCS-ELM data processing characteristics, the concentration of other pollutants can be predicted only by simple transformation of the columns in the data matrix to avoid a complicated adjustment process in the meteorological equation. Finally, the prediction accuracy of the multivariate linear regression model is positively correlated with the number of input parameters. Compare with that, HCCS-ELM is independent of the number of input parameters. So that HCCS-ELM can reduce the number of input parameters while maintaining a high prediction accuracy.

\section{Conclusion}

In areas such as Lanzhou where $\mathrm{PM}_{2.5}$ is high, the monitoring and control of $\mathrm{PM}_{2.5}$ concentrations are very important. This paper investigates HCCSELM model to predict the $\mathrm{PM}_{2.5}$ hourly and daily concentration in Lanzhou. Firstly, the hyperchaotic system generates chaotic sequences, and the values of chaotic sequences are randomly chosen instead of Lévy flights for iteration to improve the CS algorithm. Subsequently, the improved CS algorithm is used to generate the number of hidden neurons of the ELM. Moreover, considering the convergence phenomenon of chaos, hyperchaotic sequences are used to generate the input-to-hidden weight matrix and the hidden layer threshold matrix of the ELM. Finally, according to the input $\mathrm{PM}_{2.5}$ daily concentration or hourly concentration train data, the predicted result of the daily concentration or hourly concentration is obtained. Research on the experimental data obtained from four monitoring stations in Lanzhou shows that the proposed HCCSELM model can effectively predict the $\mathrm{PM}_{2.5}$ hourly and daily concentration, which is significantly better than the traditional neural network and regression 
statistical model. It is greatly significant to pollution control, and the addition of hyperchaotic system provides a new idea for improving neural networks.

\section{Experiment Platform}

CPU Intel i7-8700, GPU Nvidia RTX2060, RAM 16GB

\section{Data Source}

Meteorological data: http://data.cma.cn/

Pollutant data: http://www.mee.gov.cn/

\section{Acknowledgments}

This work was supported in part by the Fundamental Research Funds for the Central Universities (Grant No.lzujbky-2019) and The Natural Science Foundation of Gansu Province (20JR10RA606)

\section{References}

[1] Berrocal, V.J., Guan, Y., Muyskens, A., Wang, H., Reich, B.J., Mulholland, J.A., Chang, H.H.: A comparison of statistical and machine learning methods for creating national daily maps of ambient $\mathrm{PM}_{2.5}$ concentration. Atmospheric Environment 222, 117130 (2020). https://doi.org/10. 1016/j.atmosenv.2019.117130

[2] Ventura, L.M.B., de Oliveira Pinto, F., Soares, L.M., Luna, A.S., Gioda, A.: Forecast of daily $\mathrm{PM}_{2.5}$ concentrations applying artificial neural networks and holt-winters models. Air Quality, Atmosphere \& Health 12(3), 317-325 (2019). https://doi.org/10.1007/s11869-018-00660-x

[3] Kow, P.-Y., Wang, Y.-S., Zhou, Y., Kao, I.-F., Issermann, M., Chang, L.-C., Chang, F.-J.: Seamless integration of convolutional and backpropagation neural networks for regional multi-step-ahead $\mathrm{PM}_{2.5}$ forecasting. Journal of Cleaner Production 261, 121285 (2020). https://doi.org/ 10.1016/j.jclepro.2020.121285

[4] Park, Y., Kwon, B., Heo, J., Hu, X., Liu, Y., Moon, T.: Estimating PM 2.5 concentration of the conterminous united states via interpretable convolutional neural networks. Environmental Pollution 256, 113395 (2020). https://doi.org/10.1016/j.envpol.2019.113395

[5] Qiao, J., He, Z., Du, S.: Prediction of $\mathrm{PM}_{2.5}$ concentration based on weighted bagging and image contrast-sensitive features. Stochastic Environmental Research and Risk Assessment 34, 561-573 (2020). https: //doi.org/10.1007/s00477-020-01787-z 
[6] Li, K., Ma, J., Li, H., Han, Y., Yue, X., Chen, Z., Yang, J.: Discern depth under foul weather: Estimate $\mathrm{PM}_{2.5}$ for depth inference. IEEE Transactions on Industrial Informatics 16(6), 3918-3927 (2020). https: //doi.org/10.1109/tii.2019.2943631

[7] Zhou, Y., Chang, L.-C., Chang, F.-J.: Explore a multivariate bayesian uncertainty processor driven by artificial neural networks for probabilistic $\mathrm{PM}_{2.5}$ forecasting. Science of The Total Environment 711, 134792 (2020). https://doi.org/10.1016/j.scitotenv.2019.134792

[8] Wang, Y., Wang, J., Li, Z.: A novel hybrid air quality early-warning system based on phase-space reconstruction and multi-objective optimization: A case study in china. Journal of Cleaner Production 260, 121027 (2020). https://doi.org/10.1016/j.jclepro.2020.121027

[9] Xu, Y., Huang, Y., Guo, Z.: Influence of aod remotely sensed products, meteorological parameters, and aod- $\mathrm{PM}_{2.5}$ models on the $\mathrm{PM}_{2.5}$ estimation. Stochastic Environmental Research and Risk Assessment 35, 893-908 (2021). https://doi.org/10.1007/s00477-020-01941-7

[10] Wang, Z., Chen, L., Ding, Z., Chen, H.: An enhanced interval $\mathrm{PM}_{2.5}$ concentration forecasting model based on BEMD and MLPI with influencing factors. Atmospheric Environment 223, 117200 (2020). https: //doi.org/10.1016/j.atmosenv.2019.117200

[11] Jin, X.-B., Yang, N.-X., Wang, X.-Y., Bai, Y.-T., Su, T.-L., Kong, J.L.: Deep hybrid model based on EMD with classification by frequency characteristics for long-term air quality prediction. Mathematics 8(2), 214 (2020). https://doi.org/10.3390/math8020214

[12] Schraufnagel, D.E., Balmes, J.R., Cowl, C.T., Matteis, S.D., Jung, S.-H., Mortimer, K., Perez-Padilla, R., Rice, M.B., Riojas-Rodriguez, H., Sood, A., Thurston, G.D., To, T., Vanker, A., Wuebbles, D.J.: Air pollution and noncommunicable diseases. Chest 155(2), 409-416 (2019). https:// doi.org/10.1016/j.chest.2018.10.042

[13] Xiong, J., Yao, R., Wang, W., Yu, W., Li, B.: A spatial-and-temporalbased method for rapid particle concentration estimations in an urban environment. Journal of Cleaner Production 256, 120331 (2020). https: //doi.org/10.1016/j.jclepro.2020.120331

[14] Zhao, Z., Qin, J., He, Z., Li, H., Yang, Y., Zhang, R.: Combining forward with recurrent neural networks for hourly air quality prediction in Northwest of china. Environmental Science and Pollution Research (2020). https://doi.org/10.1007/s11356-020-08948-1

[15] Wang, X., Yuan, J., Wang, B.: Prediction and analysis of $\mathrm{PM}_{2.5}$ in fuling 
district of chongqing by artificial neural network. Neural Computing and Applications (2020). https://doi.org/10.1007/s00521-020-04962-z

[16] Tong, X., Ho, J.M.W., Li, Z., Lui, K.-H., Kwok, T.C.Y., Tsoi, K.K.F., Ho, K.F.: Prediction model for air particulate matter levels in the households of elderly individuals in hong kong. Science of The Total Environment 717, 135323 (2020). https://doi.org/10.1016/j.scitotenv.2019.135323

[17] Xu, Y., Liu, H.: Spatial ensemble prediction of hourly $\mathrm{PM}_{2.5}$ concentrations around beijing railway station in china. Air Quality, Atmosphere \& Health 13(5), 563-573 (2020). https://doi.org/10.1007/ s11869-020-00817-7

[18] Zhang, B., Zhang, H., Zhao, G., Lian, J.: Constructing a PM $\mathrm{PM}_{2.5}$ concentration prediction model by combining auto-encoder with bi-LSTM neural networks. Environmental Modelling \& Software 124, 104600 (2020). https://doi.org/10.1016/j.envsoft.2019.104600

[19] Pak, U., Ma, J., Ryu, U., Ryom, K., Juhyok, U., Pak, K., Pak, C.: Deep learning-based $\mathrm{PM}_{2.5}$ prediction considering the spatiotemporal correlations: A case study of beijing, china. Science of The Total Environment 699, 133561 (2020). https://doi.org/10.1016/j.scitotenv.2019.07.367

[20] Feng, L., Li, Y., Wang, Y., Du, Q.: Estimating hourly and continuous ground-level $\mathrm{PM}_{2.5}$ concentrations using an ensemble learning algorithm: The ST-stacking model. Atmospheric Environment 223, 117242 (2020). https://doi.org/10.1016/j.atmosenv.2019.117242

[21] Li, P., Du, J., Li, S., Zheng, Y., Jia, B.: The synchronization of n cascadecoupled chaotic systems. Complexity 2019, 1-10 (2019). https://doi.org/ $10.1155 / 2019 / 2709820$

[22] Jia, B., Dong, R., Du, J.: Ozone concentrations prediction in lanzhou, china, using chaotic artificial neural network. Chemometrics and Intelligent Laboratory Systems 204, 104098 (2020). https://doi.org/10.1016/j. chemolab.2020.104098

[23] Sharma, E., Deo, R.C., Prasad, R., Parisi, A.V.: A hybrid air quality earlywarning framework: An hourly forecasting model with online sequential extreme learning machines and empirical mode decomposition algorithms. Science of The Total Environment 709, 135934 (2020). https://doi.org/ 10.1016/j.scitotenv.2019.135934

[24] Feng, R.: Investigating wintertime air pollution in hangzhou, china. Air Quality, Atmosphere \& Health 13(3), 321-328 (2020). https://doi.org/10. 1007/s11869-020-00794-x 
[25] Barmpadimos, I., Keller, J., Oderbolz, D., Hueglin, C., Prévôt, A.S.H.: One decade of parallel fine $\left(\mathrm{PM}_{2.5}\right)$ and coarse $\left(\mathrm{PM}_{10}-\mathrm{PM}_{2.5}\right)$ particulate matter measurements in europe: trends and variability. Atmospheric Chemistry and Physics 12(7), 3189-3203 (2012). https://doi.org/10.5194/ acp-12-3189-2012

[26] Lou, C., Liu, H., Li, Y., Peng, Y., Wang, J., Dai, L.: Relationships of relative humidity with $\mathrm{PM}_{2.5}$ and $\mathrm{PM}_{10}$ in the yangtze river delta, china. Environmental Monitoring and Assessment 189(11) (2017). https://doi. org/10.1007/s10661-017-6281-z

[27] Li, P., Du, J., Li, S., Zheng, Y.: Modulus synchronization of a novel hyperchaotic real system and its corresponding complex system. IEEE Access 7, 109577-109584 (2019). https://doi.org/10.1109/access.2019.2934120

[28] Yang, X.-S., Deb, S.: Cuckoo search via levy flights. In: 2009 World Congress on Nature \& Biologically Inspired Computing (NaBIC), p. 210. IEEE, ??? (2009). https://doi.org/10.1109/nabic.2009.5393690

[29] Gandomi, A.H., Yang, X.-S., Alavi, A.H.: Cuckoo search algorithm: a metaheuristic approach to solve structural optimization problems. Engineering with Computers 29(1), 17-35 (2011). https://doi.org/10.1007/ s00366-011-0241-y

[30] Huang, G.-B., Zhu, Q.-Y., Siew, C.-K.: Extreme learning machine: Theory and applications. Neurocomputing 70(1-3), 489-501 (2006). https://doi. org/10.1016/j.neucom.2005.12.126

[31] Amanollahi, J., Ausati, S.: $\mathrm{PM}_{2.5}$ concentration forecasting using ANFIS, EEMD-GRNN, MLP, and MLR models: a case study of tehran, iran. Air Quality, Atmosphere \& Health 13(2), 161-171 (2019). https://doi.org/10. 1007/s11869-019-00779-5 